удК 532.529

$1986,35,2$

B. БАРАБАНЩИКОВ, В. ГЕНДРИКСОН, А. КАРТУШИНСКИИ

\title{
ТЕПЛООБМЕН В УСЛОВИЯХ УСКОРЕННОГО ДВИЖЕНИЯ КАПЕЛЬ В НЕИЗОТЕРМИЧЕСКОМ ПОЛЕ
}

\author{
(Представил И. Эпик)
}

Для охлаждения потока отработавших газов некоторых энергетических установок (снижения температуры выпускных газов) используется впрыск воды в газовыпускные устройства. Решение задачи охлаждения потока отработавших газов, включая вопросы газодинамики и теплообмена с учетом иопарения в двухфазном полидисперсном потоке типа «газ-капли», требует проведения теоретических и экспериментальных исследований. Вопросы испарения капель достаточно подробно изучены и представлены в ряде монографий $\left[{ }^{1,2}\right]$ и других материалах $\left[{ }^{3}\right]$. При этом в монографии М. Е. Дейча и Г. А. Филиппова, в частности, проведено исследование влияния противодавления на развитие паро-

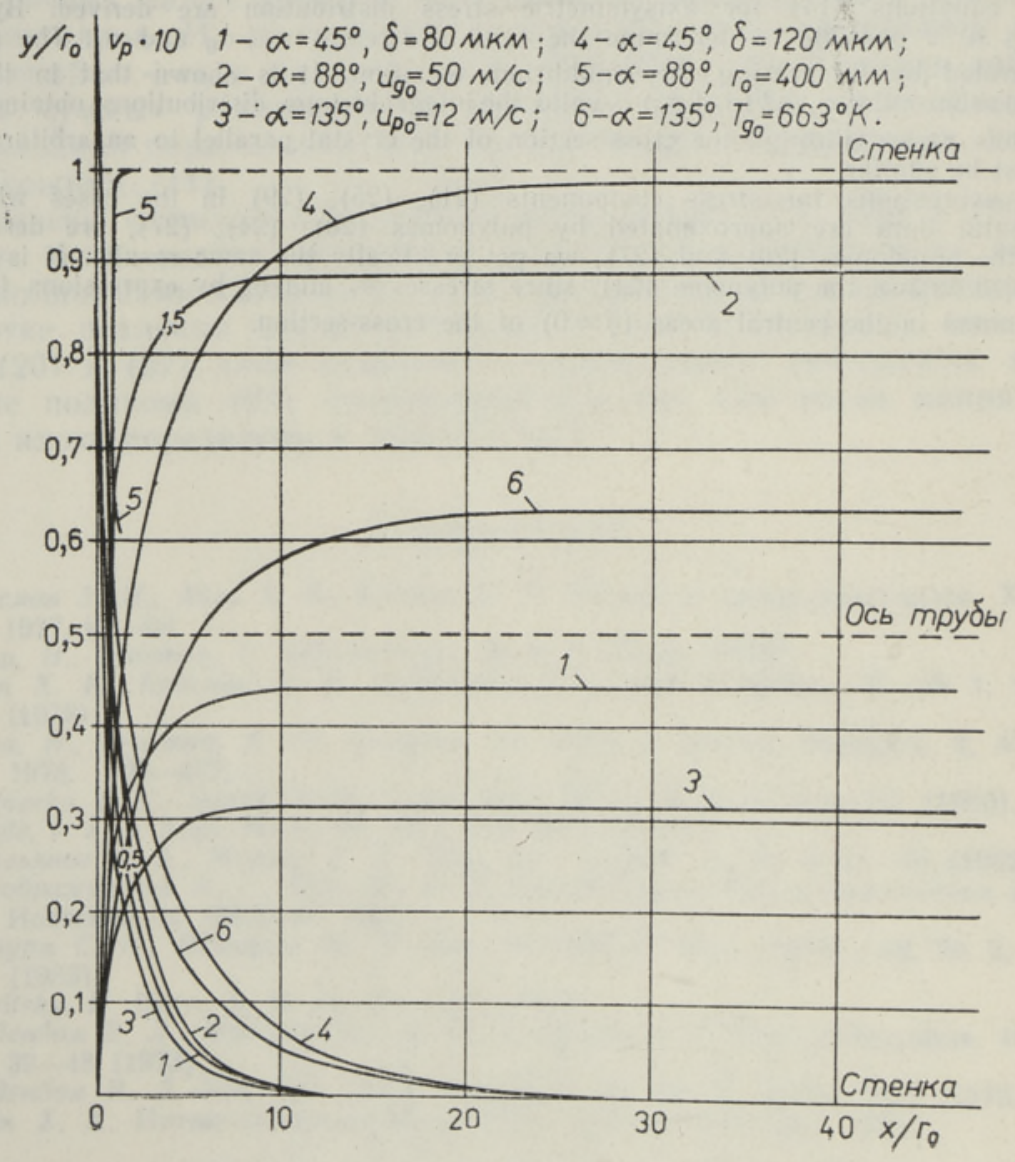

Рис. 1 . 
содержания в движущейся испаряющейся жидкости в горизонтальных цилиндрических каналах. В настоящей работе рассмотрены процессы теплообмена и иопарения в их единой последовательности в условиях, когда капли движутся ускоренно по криволинейным траекториям.

Решалась стационарная одномерная задача. Ускорение примеси осуществлялось под действием их начального импульса и силы сопротивления, записываемой в стоксовом приближении. Задача разбнвалась на два этапа. На первом определялись условия, при которых вводимые в поток под различными углами капли с ненулевым начальным импульсом при своем движении не сталкивались со стенками трубы (в противном случае не достигался бы максимальный отбор тепла у теплоносителя (газа)). Искомые траектории и поперечные скорости капель при трех углах ввода $\left(45^{\circ}, 90^{\circ}, 135^{\circ}\right)$ определялись из уравнений (везде безразмерная форма)

$$
\begin{aligned}
& u_{p} \frac{d u_{p}}{d x}=\frac{9 \varrho}{\varrho p} \frac{\left(1-u_{p}\right)}{\bar{\delta}^{2} \mathrm{Re}}, \\
& u_{p} \frac{d v_{p}}{d x}=\frac{9 \varrho}{\varrho p} \frac{\left(1-v_{p}\right)}{\bar{\delta}^{2} \mathrm{Re}},
\end{aligned}
$$

где $u_{p}$ и $v_{p}$ - осевая и радиальная скорости капель, $\bar{\delta}$ - относительный диаметр капли, равный $\bar{\delta}=\delta / d$; где $\delta$ - диаметр капли, $d-$ диаметр канала; $\varrho, \varrho p$ - физические плотности газа и капли; $\mathrm{Re}-$ число Рейнольдса канала.

Решение уравнений с начальными условиями

$$
\left.u_{p}\right|_{x=0}=0,24 \cos \alpha,\left.\quad v_{p}\right|_{x=0}=0,24 \sin \alpha
$$

осуществлялось методом Рунге-Кутта. Как показывают результаты, представленные на рис. 1 , с ростом угла ввода при $\delta=$ const внедрение капель в толщу потока по длине канала растет (кривые 1 и 2 для частиц $\delta=80$ мкм или кривые 4 и 5 для частиц $\delta=120$ мкм). Это объясняется менее градиентным затуханием радиальной скорости $v_{p}$ с ростом угла ввода капель в поток. Наилучшие результаты получаются

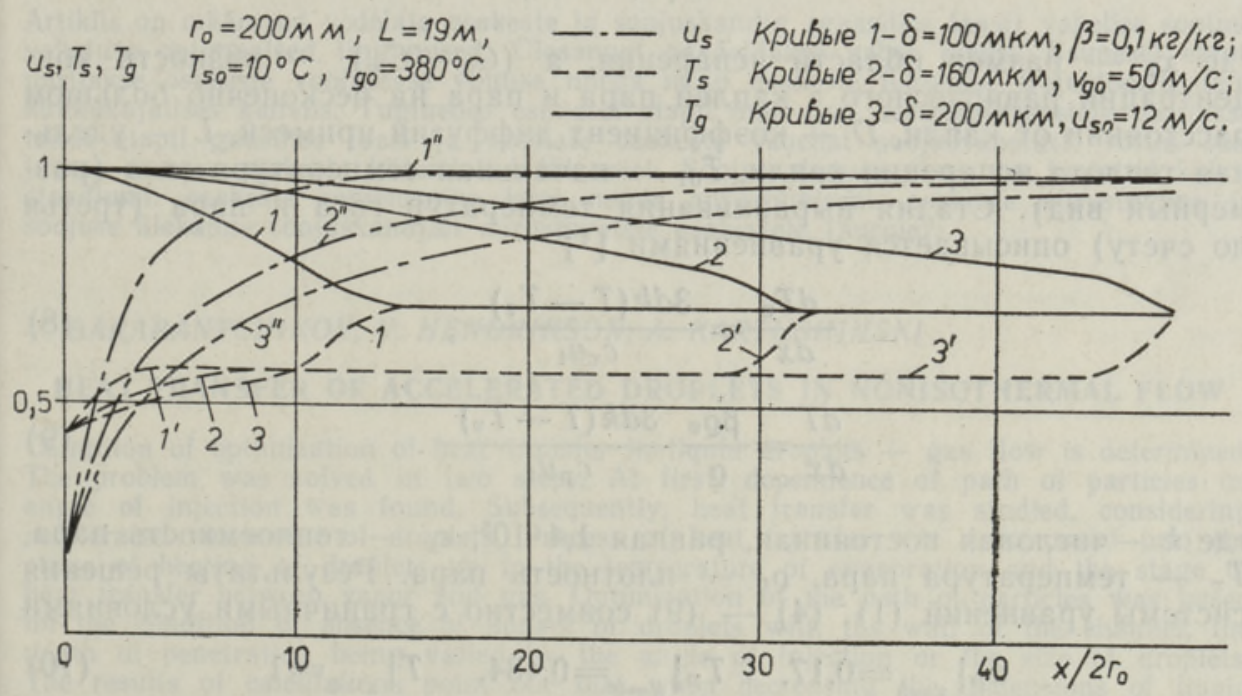

Рис. 2. 
при вводе примеси против потока ( $\alpha=135^{\circ}$; кривые 3,6 для частиц $\delta=80$ и 120 мкм соответственно). Аналогичная тенденция прослеживается при анализе влияния размера капель (при $\alpha=$ const) на степень глубины их внедрения в поток, а именно, с ростом размера частиц растет глубина внедрения. Вывод: во избежание столкновений капель со стенками трубы следует или вводить мелкую примесь, или вводить примесь в поток под наименьшим углом.

Описание процессов теплообмена капель с газовым потоком проводится на втором этапе решения задачи. При этом, основываясь на предыдущем исследовании, капли внедряются в поток горизонтально. Процесс теплообмена капель с окружающим их газом схематично раскладывается на три стадии. На первой - капли $\left(t_{p_{0}}=10^{\circ} \mathrm{C}\right)$, разгоняясь в поле высокотемпературного газового потока $\left(t_{0}=380^{\circ} \mathrm{C}\right)$, нагреваются до температуры испарения $\left(t_{p} \rightarrow 100^{\circ} \mathrm{C}\right)$; температура носителя при этом понижается. Уравнения теплообмена на этой стадии, согласно $\left[{ }^{4}\right]$, выглядят следующим образом

$$
\begin{aligned}
u_{p} \frac{d T_{p}}{d x} & =\frac{6 \varkappa\left(T-T_{p}\right)}{\delta c_{p} \varrho p u_{1} \bar{\delta}}, \\
\frac{d T}{d x} & =\frac{6 \beta \varkappa\left(T_{p}-T\right)}{\delta c_{\varrho p} u_{1} \bar{\delta}} .
\end{aligned}
$$

где $T, T_{p}$ - температуры газа и капель, $c, c_{p}$ - теплоемкости газа и капли, $x-$ коэффициент теплопроводности газовой фазы, $\beta-$ массовая концентрация примеси, $u_{1}$ - скорость газа (размерный вид). Вторая стадия - стадия испарения капель. При этом понижение температуры несущей среды в диффузионном приближении описывается, согласно $\left[{ }^{5}\right]$, уравнениями

$$
\begin{gathered}
\frac{d T}{d x}=-\frac{6 \beta D L\left(C_{0}-C_{\infty}\right)}{\delta c_{\varrho} u_{1} T_{01} \bar{\delta}}, \\
x_{2}=\frac{\delta u_{p} u_{1} \bar{\delta} u_{p}}{4 D\left(C_{0}-C_{\infty}\right)},
\end{gathered}
$$

где $x_{2}$ - размер области испарения, а $\left(C_{0}-C_{\infty}\right)-$ разность концентраций равновесного с каплей пара и пара на бесконечно большом расстоянии от капли, $D-$ коэффициент диффузии примеси, $L-$ удельная теплота испарения капли, $T_{01}$ - начальная температура газа (размерный вид). Стадия выравнивания температур газа и пара (третья по счету) описывается уравнениями [ $\left.{ }^{4}\right]$

$$
\begin{gathered}
\frac{d T_{v}}{d x}=\frac{3 d k\left(T-T_{v}\right)}{c_{v} u_{1}}, \\
\frac{d T}{d x}=\frac{\beta \varrho v}{\varrho} \frac{3 d k\left(T-T_{v}\right)}{c_{p} u_{1}} .
\end{gathered}
$$

где $k$-числовая постоянная, равная $1,4 \cdot 10^{5}, c_{v}$ - теплоемкость пара, $T_{v}-$ температура пара, $\varrho_{v}-$ плотность пара. Результаты решения системы уравнений (1), (4) - (9) совместно с граничными условиями

$$
\left.u_{p}\right|_{x=0}=0,17,\left.\quad T_{p}\right|_{x=0}=0,434,\left.\quad T\right|_{x=0}=1
$$

в виде осевых распределений скоростей и температур частиц и темпее- 
ратур газа при различных режимных условиях $(\delta=\mathrm{var})$ приведены на рис. 2. Для самой мелкой фракции капель $(\delta=100$ мкм) стадия нагрева до температуры кипения характеризуется более градиентным распределением температуры частиц (кривая 1) и температуры несущей фазы (кривая $1^{\prime}$ ), а стадия испарения с характерной горизонтальной площадкой для кривой $T_{p}=T_{p}(x)$ - наименьшей продолжительностью (размером области) по сравнению с областью испарения для более крупных капель $(\delta=160$ мкм кривая 2 и $\delta=200$ мкм кривая 3$)$. Лишь на последней стадии теплообмена градиент температур капель и газа не зависит от свойств инжектируемой в поток дисперсной жидкостной фазы (диаметра и физической плотности капель). Однако выход на равновесную температуру потока $\left(T_{p}=T\right)$ на конечной стадии теплообмена для мелкой фракции капель наступает раньше (кривые $1,1^{\prime}$ и $1^{\prime \prime}$ ), что позволяет использовать данную фракцию для оптимального отбора тепла у теплоносителя.

\title{
Л ИТЕРАТУРА
}

1. Дейч М. Е., Филиппов Г. А. Газодинамика двухфазных сред. М., Энергоиздат, 1981.

2. Салтанов Г. А. Неравновесные и нестационарные процессы в газодинамике однофазных и двухфазных сред. М., «Наука», 1979.

3. Кутателадзе С. С. Теплообмен и гидродинамика при кипении и конденсации. Мат. XXI Сиб. теплофизич. семинара. Ин-т теплофизики СО АН СССР, Новосибирск, 1982.

4. Соy С. Гидродинамика многофазных систем. М., «Мир», 1971.

5. Фукс Н. А. Испарение и рост капель в газообразной среде. М., Изд-во АН СССР, 1958.

Центральный научно-исследовательский институт им. акад. Крылова

Институт термофизики и электрофизики Академии наук Эстонской ССР

Поступила в редакцию $22 /$ II 1985

\author{
V. BARABANTSIKOV, V. HENDRIKSON, A. KARTUSINSKI
}

\section{SOOJUSULEKANNE OSAKESTE KIIRENDATUD LIIKUMISEL MITTEISOTERMILISES VÄLJAS}

Artiklis on määratud vedelate osakeste ja soojuskandja (gaasilise faasi) vahelise soojusvahetuse optimaalsed tingimused. Olesannet vaadeldakse kahes etapis. Esimesel etapil uuritakse osakeste voolusesse viimise nurga mōju osakeste liikumise trajektoorile ristkülikukujulises kanalis. Tuginedes esimesel etapil määratud seaduspärasustele uuritakse teisel etapil gaasilise faasi ja vedelate osakeste vahelist soojusvahetust, võttes seejuures arvesse osakeste kiirendatud liikumist. Soojusvahetuse etapp on jaotatud kolme staadiumi: osakeste soojenemine kuni nende aurustumiseni, osakeste aurustumine ja soojuse ülekanne soojuskandjalt aurustuvatele osakestele (aurule).

\section{BARABANTCHIKOV, V. HENDRIKSON, A. KARTUSHINSKI}

\section{HEAT TRANSFER OF ACCELERATED DROPLETS IN NONISOTHERMAL FLOW}

Condition of optimization of heat transfer in liquid droplets - gas flow is determined. The problem was solved in two steps. At first, dependence of path of particles on angle of injection was found. Subsequently, heat transfer was studied, considering accelerated movement of droplets. Process of heat transfer was decomposed into the stage of heating of droplets up to the temperature of evaporation and the stage of heat transfer between vapor and gas. Optimization of the path of particles was based on the condition of absence of impact of droplets with the wall of the channel, the depth of penetration being varied by the angle of injection or the size of droplets. The results of calculations point out that when decreasing the dimensions of liquid droplets, the state of thermal equilibrium is reached earlier; thus one can use droplets of small size to enhance heat transfer. 